\title{
Modern day management of a unilateral pleural effusion
}

\author{
Authors: Dana Li, ${ }^{A}$ Syed Ajmal, ${ }^{B}$ Muhammad Tufail ${ }^{C}$ and Rakesh K Panchal ${ }^{D}$
}

Over $\mathbf{5 0}$ systemic conditions may affect the pleura and, thus, unilateral pleural effusions may present for a variety of reasons. Investigating the cause is essential to providing appropriate management. Various pleural interventions are available in current practice, but have varying diagnostic sensitivity. It is, therefore, vital to consider the intervention with the highest diagnostic yield appropriate to the particular clinical situation. The diagnostic pathway in unilateral pleural effusion is increasingly outpatient based, avoiding hospitalisation, which is particularly relevant with the recent COVID-19 pandemic.

\section{Introduction: case presentation}

\begin{abstract}
A 67-year-old man with a background of atrial fibrillation on edoxaban presented with a 4-week history of worsening breathlessness. There is a 40-pack-year smoking history with previous asbestos exposure as a builder. The World Health Organization performance status is 0 and the general practitioner arranged a chest X-ray (CXR; Fig 1).
\end{abstract}

\section{What is the cause of this patient's symptoms?}

This patient has a unilateral moderate sized right pleural effusion.

Some patients with pleural effusion may have minimal symptoms, but others may experience breathlessness with reduced exercise capacity. This can be associated with normal or reduced oxygen saturation. The physiology of breathlessness associated with pleural effusion is not well understood but it is likely to be contributed to by reduced ventilatory capacity and gas exchange as well as by abnormal movement of the respiratory muscles with reduced expansion of the thoracic cage and splinting of the diaphragm., ${ }^{1,2}$

\section{What is the cause of the pleural effusion?}

Although the aetiology of this patient's pleural effusion is unknown, certain risk factors (including the smoking history and asbestos exposure) would suggest that a malignant pleural effusion is most likely. Ipsilateral chest pain may suggest malignant mesothelioma.

Authors: ${ }^{A}$ interventional pulmonology fellow, Glenfield Hospital, Leicester, UK; ${ }^{\text {B }}$ specialist registrar in respiratory medicine, Glenfield Hospital, Leicester, UK; ${ }^{C}$ consultant respiratory physician and honorary senior lecturer, Glenfield Hospital, Leicester, UK; ${ }^{\mathrm{D}}$ consultant respiratory physician and honorary associate professor, University Hospitals of Leicester NHS Trust, Leicester, UK and Institute for Lung Health, Leicester, UK

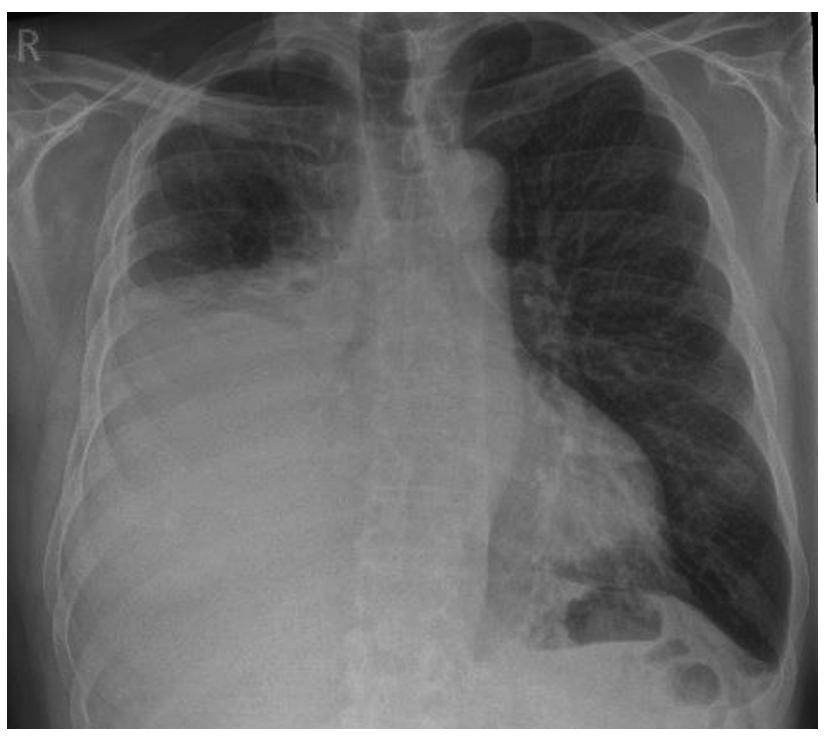

Fig 1. Initial chest X-ray.

\section{Key points}

Patients with pleural effusions may present with breathlessness due to impaired gas exchange, reduced ventilatory capacity and abnormal movement of the thoracic cage and diaphragm.

Investigation of a unilateral pleural effusion should be carefully considered, taking into account the likely underlying aetiology, the patient's clinical condition and the need for further investigation and/or intervention.

Thoracic ultrasound is essential prior to all interventions for pleural effusion and should be performed by trained operators.

Local anaesthetic thoracoscopy aids diagnosis in exudative pleural effusions of unknown aetiology.

An indwelling pleural catheter may be a useful first-line intervention in suspected or confirmed cases of malignant pleural effusion.

KEYWORDS: pleural effusion, thoracocentesis, intercostal chest drain, thoracoscopy, indwelling pleural catheter

DOI: 10.7861/clinmed.2021-0617 
Table 1. Common causes of exudative and transudative pleural effusions

\begin{tabular}{|c|c|}
\hline Exudate & Transudate \\
\hline \multirow{2}{*}{$\begin{array}{l}\text { Infection (bacterial, tuberculosis and } \\
\text { parasitic) }\end{array}$} & Congestive cardiac failure \\
\hline & Liver cirrhosis \\
\hline \multirow{2}{*}{$\begin{array}{l}\text { Malignancy (mesothelioma, lung } \\
\text { and breast) }\end{array}$} & Chronic renal failure \\
\hline & Hypoalbuminaemia \\
\hline \multicolumn{2}{|l|}{$\begin{array}{l}\text { Connective tissue disease } \\
\text { (rheumatoid arthritis) }\end{array}$} \\
\hline \multicolumn{2}{|l|}{ Drug-induced } \\
\hline \multicolumn{2}{|l|}{$\begin{array}{l}\text { Inflammatory conditions } \\
\text { (eg pulmonary infarction) }\end{array}$} \\
\hline \multicolumn{2}{|l|}{ Hypothyroidism } \\
\hline \multicolumn{2}{|l|}{ Acute pancreatitis } \\
\hline Chylothorax & \\
\hline
\end{tabular}

Pleural effusions are classified by their biochemical properties into exudative and transudative effusions but can also be due to blood, pus and chyle (Table 1). ${ }^{3}$ Applying Light's criteria may be helpful to diagnose an exudative effusion (Box 1). ${ }^{4}$ However, $25 \%$ of transudates are misclassified as exudative effusions and, therefore, calculating the serum to pleural fluid albumin gradient may be necessary to avoid misdiagnosis. ${ }^{4}$ Exudative effusions are commonly caused by infection, malignancy and inflammatory disorders, such as rheumatoid arthritis. Malignant pleural effusions (MPE), more common in lung and breast malignancies, affect up to $15 \%$ patients with cancer. ${ }^{5}$ Transudative effusions, often bilateral, are usually caused by an imbalance in hydrostatic and oncotic pressure and are associated with cardiac, renal or hepatic failure. Non-malignant pleural effusions have been shown to carry high 1-year mortality rates. ${ }^{5}$ Up to $30 \%$ of pleural effusions are multifactorial and therefore accurate aetiological diagnosis is essential as it directs subsequent management. ${ }^{6}$

The patient attends the ambulatory pleural clinic for assessment the following week.

\section{What is the most appropriate intervention?}

In patients with suspected undiagnosed malignant effusion, a therapeutic pleural aspiration may be performed while planning ahead for further intervention in the event of non-diagnostic pleural fluid cytology. Edoxaban was withheld for 48 hours prior to the procedure due to bleeding risk. The patient improved symptomatically following $1.1 \mathrm{~L}$ aspiration. The pleural fluid was sent for biochemical (protein, lactate dehydrogenase (LDH) and glucose), microbiological and cytological analysis.

\section{Box 1. Light's criteria for exudative effusions}

Exudative effusions will have one or more of the following.

Pleural fluid protein / serum protein $>0.5$.

Pleural fluid $\mathrm{LDH} /$ serum $\mathrm{LDH}>0.6$.

Pleural fluid LDH $>2 / 3$ serum $L D H$ upper limit of normal.

$\mathrm{LDH}=$ lactate dehydrogenase.
Intervention in pleural effusions can be performed for diagnostic and therapeutic purposes. Where the cause of an effusion is unclear, a decision on the most appropriate intervention should be considered carefully based on:

> the patient's symptoms and/or clinical condition

> whether the aetiology of the pleural effusion has been established

> whether further investigation may be required.

It should be noted that, when performing pleural procedures for fluid, it is essential to use immediate or real-time thoracic ultrasound (TUS). This followed the 2008 National Patient Safety Alert (NPSA) report that highlighted cases of death and severe harm resulting from chest drain insertion. ${ }^{7}$ The British Thoracic Society (BTS) has recently published training standards in TUS for respiratory clinicians that also addresses the provision of acute and emergency ultrasound. ${ }^{8}$ In addition, non-urgent procedures should not be performed if coagulopathy is present. The BTS advises to avoid procedures in anticoagulated patients until international normalised ratio $<1.5$; direct oral anticoagulants should be withheld for $24-48$ hours dependent on renal function and risk of bleeding, and therapeutic low-molecular weight heparin should also be withheld for 24 hours. ${ }^{9,10}$

\section{Diagnostic thoracocentesis}

Diagnostic thoracocentesis is an appropriate initial investigation in most patients. Performed under ultrasound guidance, it is a minimally invasive procedure that enables pleural fluid analysis to aid diagnosis. Pleural fluid cytology has variable diagnostic sensitivity in MPE. Previous BTS guidelines quoted a diagnostic accuracy of $60 \%$, however, a recent study found that overall pleural cytology sensitivity is approximately $45 \%-55 \% \%^{11,12}$ Diagnostic sensitivity is higher in ovarian, breast and lung (adenocarcinoma) malignancy at $95 \%, 71 \%$ and $82 \%$, respectively. In malignant mesothelioma, previous studies suggested diagnostic sensitivity between $16 \%$ and $73 \%{ }^{13,14}$ Although, Arnold et al recently showed that it was closer to $6 \% .{ }^{12}$ In addition, while BTS guidelines advise a minimum volume of $20-40 \mathrm{~mL}$ pleural fluid is required for diagnosis, it has been found that a minimum volume of $75 \mathrm{~mL}$ increases diagnostic sensitivity. ${ }^{15,16}$ Even if a diagnosis is obtained, the material may not always be sufficient for molecular markers / driver mutations, which will aid in decision making for appropriate immunotherapy or targeted molecular therapy.

\section{Therapeutic thoracocentesis}

Therapeutic thoracocentesis performed under ultrasound guidance allows for pleural fluid analysis to aid diagnosis, as well as removal of a larger volume of pleural fluid to provide symptom benefit. The procedure avoids the insertion of a chest drain and, therefore, can be performed on ambulant patients in an outpatient setting to avoid hospitalisation. If pleural fluid cytology is non-diagnostic, residual pleural fluid enables further invasive procedures to be performed including image-guided pleural biopsy or local anaesthetic thoracoscopy.

\section{Intercostal chest drain}

Intercostal chest drain (ICD) insertion performed under ultrasound guidance enables pleural fluid analysis, complete drainage of 

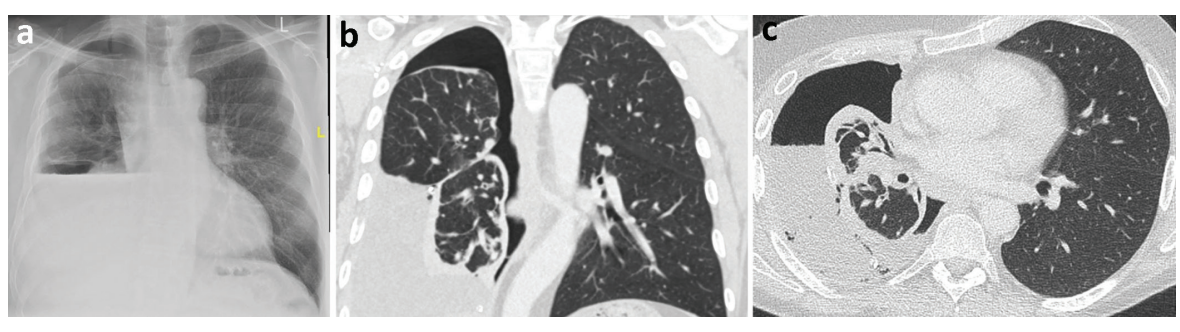

Fig 2. a) Chest X-ray post-thoracentesis demonstrating hydropneumothorax (air and fluid in the pleural cavity). b) Computed tomography of the chest, coronal view. c) Computed tomography of the chest, axial view.

the pleural effusion and administration of intrapleural agents, if required. Commonly, talc slurry pleurodesis is administered through the ICD to prevent recurrence of probable or confirmed MPE. Talc slurry pleurodesis has a failure rate of $24 \% .{ }^{17}$ This option may be appropriate for symptomatic patients with large volume pleural effusions who are non-ambulant with poor performance status where further invasive tests may not be appropriate or where the aetiology of the effusion is known.

The BTS guidelines advise caution if removing $>1.5 \mathrm{~L}$ of pleural fluid on a single occasion due to the risk of developing re-expansion pulmonary oedema (RPO), a condition that can lead to cough, chest pain and even cardiovascular instability and collapse. ${ }^{18}$ Following 16 cases of RPO between 2017-2020, an NPSA alert has highlighted the need to ensure controlled drainage following ICD insertion to avoid this potential complication. ${ }^{19}$ Clinicians should be mindful that interventions for pleural effusion, despite the use of thoracic ultrasound, should be avoided out-of-hours unless in an emergency to minimise unnecessary errors and complications.

A CXR following therapeutic thoracocentesis revealed a hydropneumothorax (Fig 2a), raising the possibility of a non-expandable lung (NEL). Pleural fluid analysis suggested an exudative effusion (protein of $40 \mathrm{~g} / \mathrm{L}$ and LDH of $620 \mathrm{IU}$ ). Cytology revealed a lymphocytic effusion with no malignant cells seen. Computed tomography of the chest (Fig 2b and 2c) demonstrated a parietal pleural rind predominantly encasing the right lower lobe, likely causing the NEL.

\section{What is the next most appropriate intervention?}

As the pleural fluid cytology was non-diagnostic, the patient proceeded to a thoracoscopy under local anaesthetic and moderate sedation, also known as local anaesthetic thoracoscopy (LAT), medical thoracoscopy or pleuroscopy.

LAT is a medical procedure, performed by respiratory physicians, involving internal examination, biopsy and/or administration of intrapleural agents within the pleural cavity. It is done under moderate sedation and local anaesthesia. The main indication for LAT is in the investigation of exudative effusions of unknown aetiology, particularly when there is no pleural target for CT-guided biopsy and pleurodesis is being considered. LAT is also useful in the diagnosis of pleural tuberculosis with a combined culture/histology sensitivity of up to $100 \%$, depending on prevalence. $^{20}$

The efficacy of LAT in the diagnosis of malignancy is as high as video-assisted thoracoscopic surgery (VATS) biopsy. ${ }^{21}$ VATS biopsies are performed by thoracic surgeons, usually under general anaesthesia, with single lung ventilation.
LAT can also be used for therapeutic indications. Pleurodesis by talc poudrage can be undertaken if the pleura appears abnormal on direct inspection. It is as effective as talc slurry in achieving pleurodesis with increased efficacy in patients with lung or breast cancer. ${ }^{16}$ LAT may also be useful in pleural infections, allowing for division of septations and adhesions and aiding in accurate chest drain placement. ${ }^{22}$

LAT can be performed using a semi-rigid thoracoscope (Fig 3a), which is of similar design to a flexible video bronchoscope or a rigid thoracoscope (Fig 3b); both yield similar diagnostic accuracy. ${ }^{23}$

The patient underwent local anaesthetic (medical) thoracoscopy. This showed extensive nodularity over the visceral and parietal pleural (Fig 4a). A decision was made to insert an indwelling pleural catheter (IPC) at the time of thoracoscopy for likely NEL (Fig 4b).

\section{What is the role of indwelling pleural catheters?}

IPCs have established themselves as an important tool in the management of pleural effusions. An IPC is a soft, flexible silicone tube with multiple fenestrations (Fig $5 \mathrm{a}$ ). It is tunnelled subcutaneously before entering the pleural cavity (Fig $5 b$ ). The external portion contains a one-way valve that connects to a closed drainage system with vacuum (Fig 5c).

IPCs were initially designed as a second-line treatment for patients who have MPE with NEL or failed pleurodesis and can remain in the pleural cavity and outlive the patient. NEL (or trapped lung) occurs when the lung cannot expand after fluid drainage, either because of endobronchial obstruction or visceral pleural encasement by tumour. ${ }^{24}$

The role of IPCs in pleural effusion management saw a paradigm shift with the publication of the TIME2 and AMPLE trials supporting their use as a first-line treatment. ${ }^{25,26}$ Depending on patient preference, IPCs may be offered as a first-line treatment in MPEs and are usually done as an elective day case procedure. They are safe and reduce length of hospital stay in patients who would traditionally have needed prolonged and often multiple admissions. IPCs have shifted the management of pleural effusions to a more outpatient-orientated setting.

The drainage of IPCs is supported by district nurses but patients are encouraged to drain and manage their own devices. Drainage frequency is commonly two to three times per week but can be tailored to meet the patient's needs. If the aim is palliation, then symptom-guided drainage may be appropriate. ${ }^{27}$ However, if the aim is to achieve rapid pleurodesis with subsequent drain removal, then daily drainage may lead to a higher rate of autopleurodesis and faster time to IPC removal. ${ }^{28}$ Autopleurodesis is defined as spontaneous pleurodesis without the use of a chemical agent. 
Fig 3. a) Semi-rigid thoracoscope. Reproduced with permission from Olympus Europa SE \& Co. b) Rigid thoracoscope.
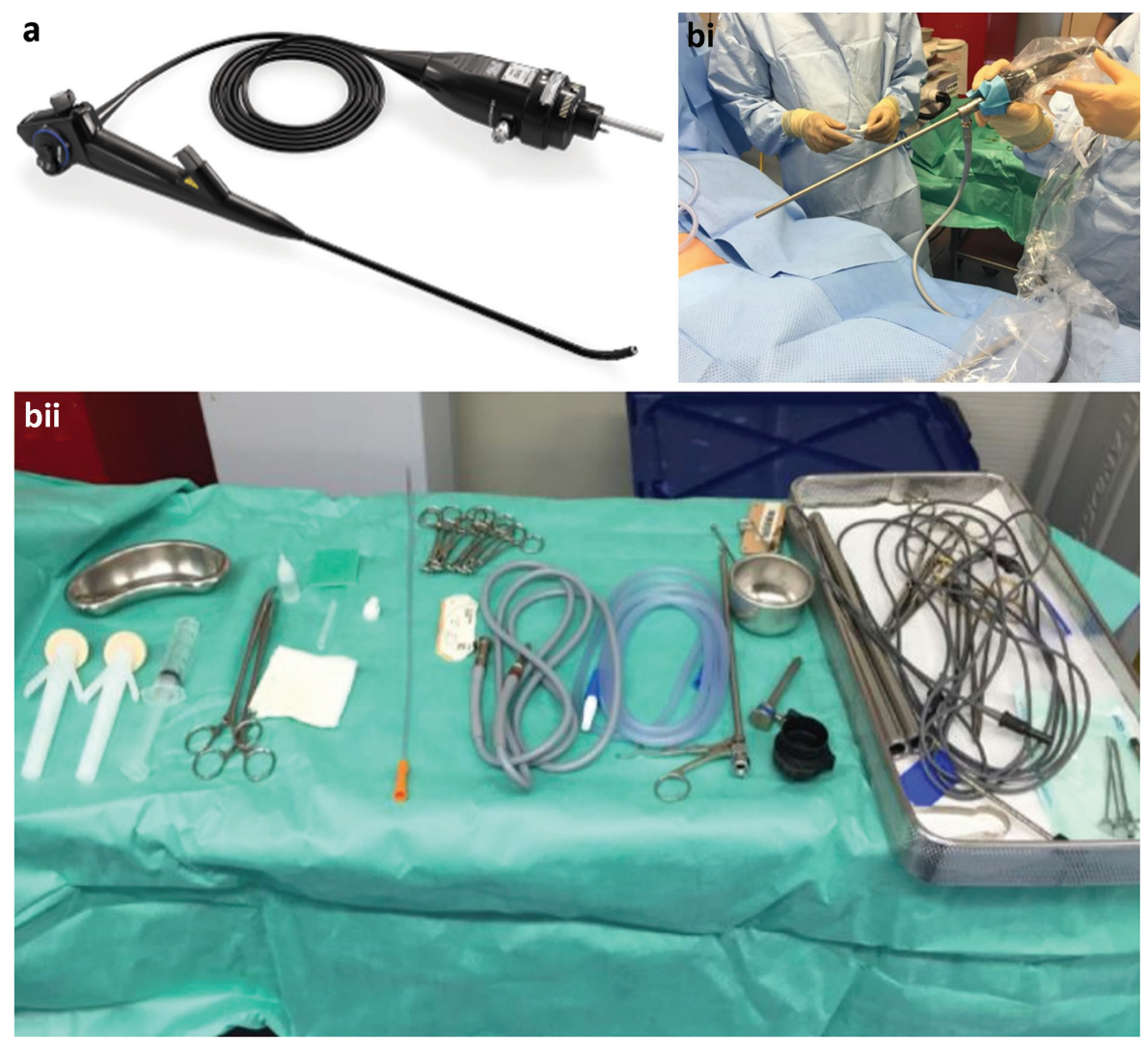

Although not the objective of IPC insertion, it can be observed in up to $51 \%$ of patients with MPE. ${ }^{25}$ Additionally, aggressive drainage with administration of talc via the IPC can result in twofold higher rates of pleurodesis compared with IPC alone. ${ }^{29}$

The role of IPCs in non-malignant pleural effusions is less well defined and needs to be tailored to the patient. The first randomised trial on refractory transudative pleural effusions concluded that IPCs did not offer superior control of breathlessness compared with as required therapeutic thoracentesis, with the IPC arm experiencing higher adverse events. ${ }^{30}$ In hepatic hydrothorax, IPCs can be offered with palliative intent to patients who have exhausted medical management and are not transplant candidates or in some patients as a bridging therapy to manage effusions prior to transplantation. ${ }^{31,32}$

As IPC use becomes more widespread, clinicians may encounter more IPC-related complications that may occur in $10 \%-20 \%$ of

Fig 4. a) Parietal pleura biopsies taken during a thoracoscopy and thoracoscopic view of a malignant nodule. b) Chest X-ray post-thoracoscopy.
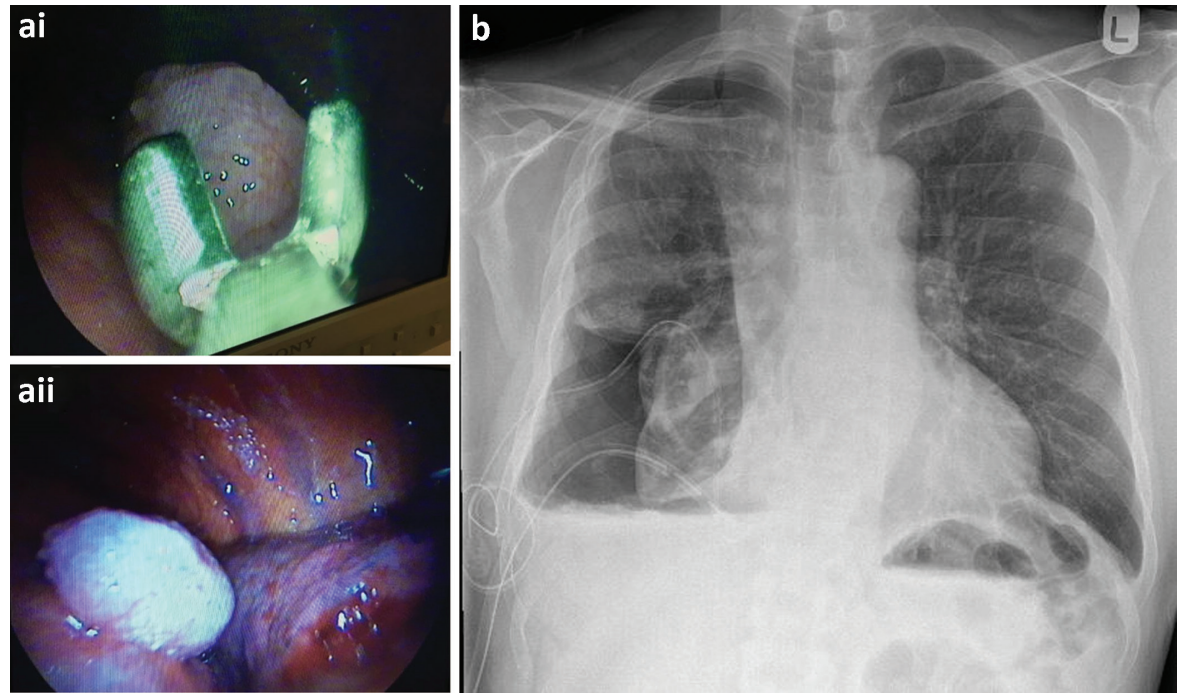

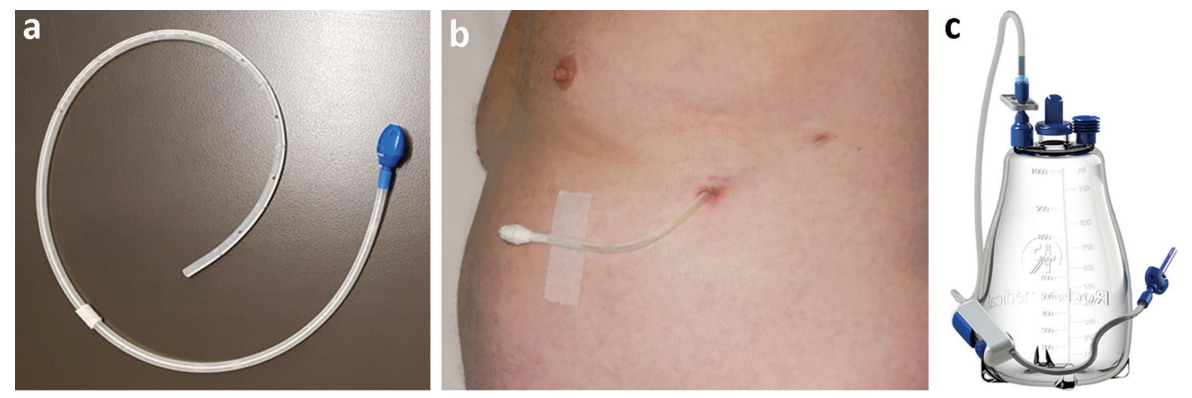

Fig 5. a) The components of an indwelling pleural catheter. b) A tunnelled indwelling pleural catheter. Reproduced with permission from Rocket Medical. c) An indwelling pleural catheter drainage system. Reproduced with permission from Rocket Medical.

patients. ${ }^{33}$ Pleural infection, catheter blockage and symptomatic loculations tend to be the most common and can be managed. ${ }^{34}$ In oncology patients, there is good evidence that there is no increased risk of IPC-related infection by chemotherapy or an immunosuppressed state. ${ }^{35,36}$

The pleural biopsies revealed malignant epithelioid mesothelioma. Following discussion in the lung multidisciplinary team meeting, the patient was referred to oncology for systemic treatment. The patient continued to drain his IPC three times weekly and subsequently achieved autopleurodesis. The IPC was removed soon after.

\section{Summary}

Investigation of a unilateral pleural effusion is a common clinical scenario faced by general and respiratory physicians alike. With the high prevalence of MPE associated with advanced-stage lung cancer and other malignancies, it is increasingly important to

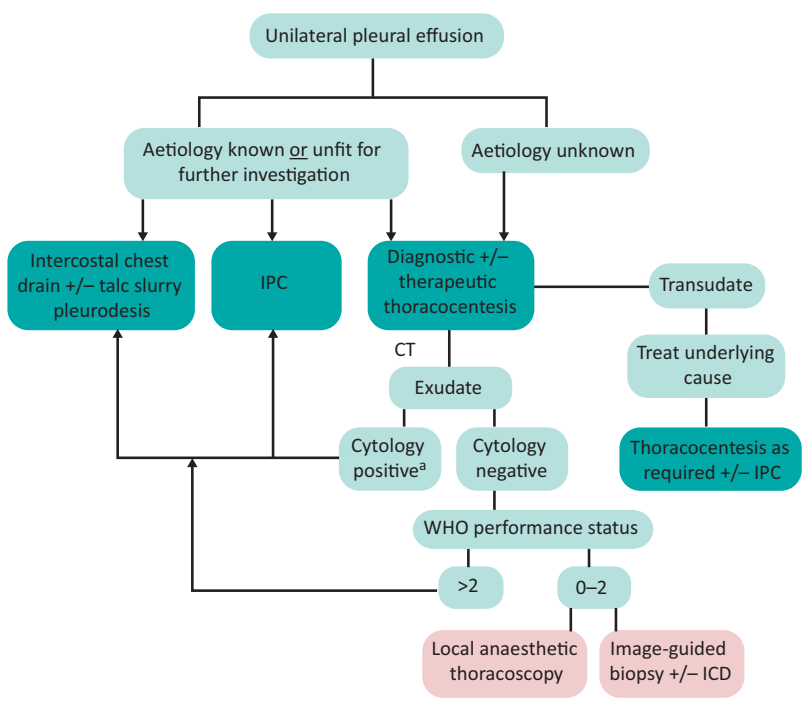

Fig 6. Pathway for investigation of a unilateral pleural effusion. ${ }^{a}$ If further tissue required for molecular testing, consider local anaesthetic thoracoscopy; $\mathrm{CT}=$ computed tomography; IPC = indwelling pleural catheter; $\mathrm{WHO}=$ World Health Organization . perform the correct, as well as most useful, investigation for the patient (Fig 6). This is particularly relevant with recent advances in oncological therapy, whereby diagnosis on pleural cytology alone may be insufficient, and supplementary pleural histology may provide additional information (including molecular analysis) to guide treatment.

As with many aspects of modern patient care, the investigation and management of patients with pleural effusions is increasingly managed in an ambulatory fashion. With the adoption of sameday emergency services and pleural clinics in many hospitals, this provides patients with streamlined, efficient and effective care. This has been particularly relevant during the COVID-19 pandemic and reduces cost and risk of hospital-associated infection to the patient. This, together with devices (such as the IPC) that have been designed specifically for patients wishing to remain at home, has allowed the emergence of a modern approach to pleural medicine.

\section{Acknowledgements}

The authors acknowledge review of the manuscript by Prof Jonathan Bennett, consultant respiratory physician and honorary professor at University Hospitals of Leicester NHS Trust and chair of the British Thoracic Society.

\section{References}

1 Thomas R, Jenkins S, Eastwood PR, Lee YCG, Singh B. Physiology of breathlessness associated with pleural effusions. Curr Opin Pulm Med 2015;21:338-45.

2 Muruganandan S, Azzopardi M, Thomas R et al. The Pleural Effusion And Symptom Evaluation (PLEASE) study of breathlessness in patients with a symptomatic pleural effusion. Eur Respir ] 2020;55:1900980.

3 Mercer RM, Corcoran JP, Porcel JM, Rahman NM, Psallidas I. Interpreting pleural fluid results. Clin Med 2019;19:213-7.

4 Light RW. The Light criteria: the beginning and why they are useful 40 years later. Clin Chest Med 2013;34:21-6.

5 Walker SP, Morley AJ, Stadon L et al. Nonmalignant pleural effusions: a prospective study of 356 consecutive unselected patients. Chest 2017;151:1099-105.

6 Bintcliffe OJ, Hooper CE, Rider I] et al. Unilateral pleural effusions with more than one apparent etiology. a prospective observational study. Ann Am Thorac Soc 2016;13:1050-6.

7 National Patient Safety Agency (NPSA). Rapid response report: risks of chest drain insertion. Reference NPSA/2008/RRR03. London: NPSA, 2008:1-10. www.npsa.nhs.uk/nrls/alerts-and-directives/ rapidrr/risks-of-chest-drain-insertion [Accessed 10 September 2021]. 
8 Stanton AE, Edey A, Evison M et al. British Thoracic Society Training Standards for Thoracic Ultrasound (TUS). BMJ Open Resp Res 2020;7:e000552.

9 Keeling D, Tait RC, Watson H, the British Committee of Standards for Haematology. Peri-operative management of anticoagulation and antiplatelet therapy. Br J Haematol 2016;175:602-13.

10 Douketis JD, Spyropoulos AC, Spencer FA et al. Perioperative management of antithrombotic therapy: Antithrombotic Therapy and Prevention of Thrombosis, 9th ed: American College of Chest Physicians Evidence-Based Clinical Practice Guidelines. Chest 2012;141(2 Suppl):e326S-50S.

11 Maskell NA, Butland RJA, Pleural Diseases Group, Standards of Care Committee, British Thoracic Society. BTS guidelines for the investigation of a unilateral pleural effusion in adults. Thorax 2003;58 Suppl 2:ii8-17.

12 Arnold DT, De Fonseka D, Perry S et al. Investigating unilateral pleural effusions: the role of cytology. Eur Respir J 2018;52:1801254.

13 Walters ], Maskell NA. Biopsy techniques for the diagnosis of mesothelioma. In: Tannapfel A (ed). Malignant mesothelioma. Springer, 2011:45-55. http://link.springer.com/10.1007/978-3-642-10862 4_4 [Accessed 15 September 2021].

14 Segal A, Sterrett GF, Frost FA et al. A diagnosis of malignant pleural mesothelioma can be made by effusion cytology: results of a 20 year audit. Pathology 2013;45:44-8.

15 Hooper C, Lee YCG, Maskell N, on behalf of the BTS Pleural Guideline Group. Investigation of a unilateral pleural effusion in adults: British Thoracic Society pleural disease guideline 2010. Thorax 2010;65(Suppl 2):ii4-17.

16 Rooper LM, Ali SZ, Olson MT. A minimum fluid volume of $75 \mathrm{~mL}$ is needed to ensure adequacy in a pleural effusion: A retrospective analysis of 2540 cases: minimum adequate pleural effusion volume. Cancer Cytopathology 2014;122:657-65.

17 Bhatnagar R, Piotrowska HEG, Laskawiec-Szkonter M et al. Effect of thoracoscopic talc poudrage vs talc slurry via chest tube on pleurodesis failure rate among patients with malignant pleural effusions: a randomized clinical trial. JAMA 2020;323:60.

18 Havelock T, Teoh R, Laws D, Gleeson F, on behalf of the BTS Pleural Disease Guideline Group. Pleural procedures and thoracic ultrasound: British Thoracic Society pleural disease guideline 2010. Thorax 2010;65(Suppl 2):i61-76.

19 National Patient Safety Agency (NPSA). Rapid response report: Deterioration due to rapid offload of pleural effusion fluid from chest drains. NatPSA/2020/008/NHSPS. London: NPSA, 2020:1-2.

20 Diacon AH, Van de Wal BW, Wyser C et al. Diagnostic tools in tuberculous pleurisy: a direct comparative study: 1. Eur Respir ] 2003:22:589-91.

21 Hansen M, Faurschou P, Clementsen P. Medical thoracoscopy, results and complications in 146 patients: a retrospective study. Respiratory Medicine 1998;92:228-32.

22 Ravaglia C, Gurioli C, Tomassetti S et al. Is medical thoracoscopy efficient in the management of multiloculated and organized thoracic empyema? Respiration 2012;84:219-24.

23 Rozman A, Camlek L, Marc-Malovrh M, Triller N, Kern I. Rigid versus semi-rigid thoracoscopy for the diagnosis of pleural disease: a randomized pilot study. Respirology 2013;18:704-10.
24 Lee YCG. Expanding knowledge on non-expandable lungs. Respirology 2020;25:238-9.

25 Davies HE, Mishra EK, Kahan BC et al. Effect of an indwelling pleural catheter vs chest tube and talc pleurodesis for relieving dyspnea in patients with malignant pleural effusion: the TIME2 randomized controlled trial. JAMA 2012;307:2383.

26 Thomas R, Fysh ETH, Smith NA et al. Effect of an indwelling pleural catheter vs talc pleurodesis on hospitalization days in patients with malignant pleural effusion: the AMPLE randomized clinical trial. JAMA 2017;318:1903.

27 Muruganandan S, Azzopardi M, Fitzgerald DB et al. Aggressive versus symptom-guided drainage of malignant pleural effusion via indwelling pleural catheters (AMPLE-2): an open-label randomised trial. Lancet Respir Med 2018;6:671-80.

28 Wahidi MM, Reddy C, Yarmus L et al. Randomized trial of pleural fluid drainage frequency in patients with malignant pleural effusions: the ASAP trial. Am J Respir Crit Care Med 2017;195: 1050-7.

29 Bhatnagar R, Keenan EK, Morley AJ et al. Outpatient talc administration by indwelling pleural catheter for malignant effusion. $N$ Engl J Med 2018:378:1313-22.

30 Walker SP, Bintcliffe O, Keenan E et al. Randomised trial of indwelling pleural catheters for refractory transudative pleural effusions. Eur Respir ] 2021;2101362.

31 Shojaee S, Rahman N, Haas K et al. Indwelling tunneled pleural catheters for refractory hepatic hydrothorax in patients with cirrhosis. Chest 2019;155:546-53.

32 Gilbert CR, Shojaee S, Maldonado F et al. How I do it: pleural interventions in the management of hepatic hydrothorax. Chest 2021;S0012-3692(21)03672-2 [Epub ahead of print].

33 Lui MMS, Thomas R, Lee YCG. Complications of indwelling pleural catheter use and their management. BMJ Open Resp Res 2016;3:e000123.

34 Chalhoub M, Saqib A, Castellano M. Indwelling pleural catheters: complications and management strategies. J Thorac Dis 2018;10:4659-66.

35 Wilshire CL, Jackson AS, Horslen LC et al. Clinical evolution of practice patterns in the management of pleural space infections: a community-based healthcare network review. Ann Am Thorac Soc 2021;18:1592-4.

36 Mekhaiel E, Kashyap R, Mullon J], Maldonado F. Infections associated with tunnelled indwelling pleural catheters in patients undergoing chemotherapy. J Bronchology Interv Pulmonol 2013:20:299-303.

Address for correspondence: Dr Dana Li, Department of Respiratory Medicine, University Hospitals of Leicester NHS Trust, Glenfield Hospital, Groby Road, Leicester LE3 9QP, UK. Email: dana.li@uhl-tr.nhs.uk Twitter: @leicschestdoc 